Meta

Journal des traducteurs

Translators' Journal

\title{
Lexicographie et linguistique : la signification du mot
}

\section{F. de Tollenaere}

Volume 18, numéro 1-2, mars 1973

Actes du deuxième colloque international de linguistique et de traduction. Montréal, 4-7 octobre 1972

URI : https://id.erudit.org/iderudit/004493ar

DOI : https://doi.org/10.7202/004493ar

Aller au sommaire du numéro

Éditeur(s)

Les Presses de l'Université de Montréal

ISSN

0026-0452 (imprimé)

1492-1421 (numérique)

Découvrir la revue

Citer cet article

de Tollenaere, F. (1973). Lexicographie et linguistique : la signification du mot. Meta, 18(1-2), 139-144. https://doi.org/10.7202/004493ar d'utilisation que vous pouvez consulter en ligne.

https://apropos.erudit.org/fr/usagers/politique-dutilisation/ 


\section{Lexicographie et linguistique: la signification du mot}

0. Qu'il me soit permis de dire un mot à propos du titre de ma communication. Si l'on ne peut pas dire que ce titre lui va comme un tablier à une vache, il est pourtant vrai que le pavillon qui couvre la marchandise puisse produire de faux espoirs. Je parlerai, bien sûr, de lexicographie historique et linguistique, mais spécialement dans le cadre d'un seul problème : celui de la signification du mot.

1. En guise d'introduction, je me bornerai à mentionner le fait que les linguistes sont souvent assez sévères dans leur critique envers les lexicographes. Heureusement pour nous autres, lexicographes, gratte-papier inoffensifs (harmless drudges) selon le mot célèbre de Samuel Johnson. Le linguiste américain Gleason a dit à propos de quelques-uns de ses collègues, que les principes qui sont à la base de leur critique ne s'appliquent que fort mal aux dictionnaires. Les exigences qu'ils formulent sont, a-t-il dit, peu raisonnables; par ce fait, ils font preuve de ne comprendre que fort mal les limitations dans lesquelles le lexicographe doit opérer (Gleason, 1962, p. 88).

La question de savoir si la critique de certains linguistes envers la discipline lexicographique se fait à tort ou à bon droit, ne nous retiendra pourtant pas dans ce qui suit. Notre exposé vise, tout au contraire, d'une façon critique une certaine conception linguistique concernant le problème de la signification.

2. Mon exposé se rapporte à la signification du mot. On a dit que la signification du mot est un objet mal défini, ou, si l'on veut, que les linguistes s'accordent mal en ce qui concerne la nature de la signification lexicale (Zgusta, 1971, p. 23). Cela ne peut cependant trop gêner le lexicographe. La phrase, voire même le mot, ne sont pas bien définis non plus, ce qui n'a jamais empêché les grammairiens de s'en occuper.

Il va de soi que le lexicographe, différemment des linguistes de l'école de Bloomfield, ne peut mettre la signification entre parenthèses, ni la considérer comme appartenant à la métalinguistique. S'il est vrai que la linguistique ne peut exister sans la signification (Allen, 1957, p. 22), il est plus vrai encore que la lexicographie ne peut se passer de la signification du mot.

Nous considérons la signification du mot comme «le rapport d'évocation réciproque qui unit le nom et le sens $\gg$ (Ullmann, 1952, 1959, p. 23). Dans cette définition on peut, si l'on veut éviter une équivoque possible, remplacer 
le terme sens par celui de concept. La conception d'Ullmann est illustrée par le triangle classique du nom, du concept et de la chose, ce modèle général de l'analyse sémantique traditionnelle. Je sais bien que ce modèle ne convient pas également bien à tous les mots. Il ne s'adapte cependant pas mal à nombre de mots lexicaux, les seuls que j'envisagerai ici dans le but de simplifier mon exposé.

3. Ma communication vise la théorie de la signification du mot qui est généralement acceptée aux Pays-Bas, et cela depuis des dizaines d'années déjà, notamment depuis l'apparition en 1935 du livre de Reichling, intitulé Het Woord. Si les publications de Reichling sont moins connues à l'étranger qu'elles ne le méritent, cela tient du fait qu'elles ont été écrites généralement en néerlandais, à l'exception de quelques articles anglais parus dans la revue Lingua, 1 (1948), 10 (1961), 11 (1962); dans For Roman Jakobson (1956), et dans les Proceedings of the Ninth International Congress of Linguists (1964).

La théorie de la signification du mot que Reichling a exposée dans son livre de 1935, se retrouve dans ses autres publications, même les plus récentes. Dans un article écrit en langue allemande sur le problème de la signification en linguistique (1963), Reichling n'a pourtant pas répété cette théorie.

Selon lui, le terme signification doit se réserver exclusivement au mot et il se refuse à parler de la signification de la phrase ou de la signification du groupe de mots. Pour ces deux derniers cas il préfère le terme contenu de la phrase, respectivement contenu du groupe de mots. Étant donné cette limitation du terme signification la théorie du linguiste hollandais peut se résumer en deux thèses :

a) La signification peut se définir comme une unité de connaissance constante, incorporée dans un certain mot; ou un certain «savoir», qui, dans la plupart des parties du discours, présente une pluralité de distinctions utilisables de manière diverse. L'unité de signification reste identique dans chaque cas où le mot est employé, même si ce que nous apprenons en employant un certain mot dans une certaine phrase peut différer énormément.

b) La polysémie du mot se définit généralement comme suit : un mot peut avoir beaucoup de significations différentes. Cela est faux. Un mot a toujours une seule signification, mais à l'intérieur de cette signification unique peuvent se présenter des distinctions très différentes (Reichling, 1961, p. 37 ; Reichling, 1965, p. 42).

Cette théorie de Reichling connut très tôt un accueil chaleureux. Dans un article paru dans les années 1941-1942, dans une revue de philosophie et de psychologie hollandaise, on estime que Reichling a purifié le mot et la langue de l'odieux d'être polysémique, c'est-à-dire d'être un moyen de communication défectueux (Stutterheim, 1941-1942, p. 194).

La théorie reichlingienne de la signification du mot semble de nos jours avoir été acceptée généralement aux Pays-Bas. Les écrits de plusieurs titulaires de chaires de linguistique générale, ainsi que des publications d'autres linguistes en font témoignage. Citons à titre d'exemple ce qu'on trouve dans un livre de linguistique générale écrit à l'usage des étudiants par le successeur de Reichling 
à la chaire de linguistique générale de l'Université d'Amsterdam. Je cite en traduisant : «La signification du mot a plusieurs facettes. Quand, dans une description synchronique d'une langue, les distinctions sémantiques d'un mot n'ont plus de relation entre elles, nous parlons de deux significations et nous considérons de tels mots comme des homonymes. Ainsi : néerl. bank «établissement de crédit» (fr. banque) et néerl. bank «siège étroit et long» (fr. banc) sont deux mots différents avec deux significations différentes et une seule forme phonétique. Par contre, école «bâtiment» et «institut» ne sont que des facettes d'un seul et même mot école » (Dik et Kooij, 1970, p. 164).

Nul ne peut se douter, me semble-t-il, de l'influence de cette théorie sémantique de Reichling aux Pays-Bas. Il m'est arrivé, en assistant à un congrès à Amsterdam, d'être regardé par certaines personnes d'un air peiné, lorsque j'eus l'audace de parler des significations du mot, c'est-à-dire, en me permettant d'employer le mot signification dans son acception ordinaire. Il n'est point douteux que la conception de Reichling concernant la signification du mot a pris aux Pays-Bas le caractère dangereux d'un dogme linguistique. Par conséquent on est parvenu à jeter un tabou sur l'expression : les significations d'un mot.

Personne ne voudra refuser à Reichling le droit d'employer le mot signification dans un sens spécial. Il y a en effet des mots nombreux qui ont un sens tout à fait extraordinaire non seulement en linguistique générale ou dans la terminologie de certains linguistes, mais dans n'importe quelle discipline scientifique. Qu'il me soit permis pourtant de formuler quelques remarques critiques.

a) D'abord la définition de la signification du mot chez Reichling, ou, si l'on veut, la signification qu'il donne au mot signification, est très vague. C'est un tout insaisissable, un conglomérat se composant bien sûr d'éléments saisissables, mais dont la somme ne se laisse point concrétiser. Cette signification de Reichling ne se rencontre en effet jamais et nulle part. C'est une abstraction psychologique, voire métaphysique, d'entités linguistiques réelles. Seules ces dernières se laissent définir. On a dit, non à tort, que la vision de Reichling fait songer à celle de la scolastique, pour laquelle le terminus conservait sa significatio dans ses différentes suppositiones (Stutterheim, 1941-1942, p. 193). Si vraiment les contenus sémantiques «meuble » et « mets, nourriture » du mot table, par exemple, constituaient une seule signification, alors il devrait être possible d'en donner une définition unique. La définition générale donnée par Reichling à la signification du mot, à savoir, "unité de connaissance constante », relie bien «meuble » et «mets», mais elle peut s'appliquer tout aussi bien au mot table, qu'aux mots mouchoir, polichinelle ou turlupin. Dès lors cette définition s'avère inopérante. Ce qu'il proclame signification unique est en réalité une chaîne de significations, dont les membres (appelés significations dans l'usage journalier) forment une continuité synchronique. L'étiquette signification est collée par le linguiste hollandais sur la collectivité des maillons d'une châ̂ne sémantique. Il a employé, à tort à mon avis, le mot signification pour désigner la chaîne sémantique synchronique.

b) La signification chez Reichling est en outre d'un caractère assez subjectif. Étant donné un point de départ synchronique cette unité de signification peut 
varier selon les sujets parlants. Cela implique que ceux-ci ne donneront pas toujours la même réponse à la question si deux mots appartiennent à la même unité de signification, c'est-à-dire, s'ils sont à considérer comme des entités différentes. La réponse sera influencée par le degré de culture générale et par la conscience linguistique du sujet parlant. Par exemple : du moment que l'on sait que la signification «espèce » du mot néerlandais slag a évolué à travers celle de "frappe (de la monnaie) », les mots slag «coup » et slag « espèce » peuvent cesser d'être considérés comme homonymes et devenir des membres d'une seule chaîne sémantique. Dans ce contexte on pourrait, par exemple, penser au mot grue "oiseau échassier» et grue « appareil de levage».

Les deux objections critiques que je viens de donner sont d'un caractère plutôt général; je veux en donner deux autres en me mettant sur le plan lexicographique.

c) Si c'est le droit du linguiste Reichling de donner aux mots signification et polysémie des acceptions inusitées et inconnues en lexicographie, on ne peut cependant point s'attendre à voir les lexicographes reprendre sa terminologie. Celui qui fait un dictionnaire ne peut point s'adapter à chaque lubie de changement terminologique de linguistes. L'acception donnée par Reichling au mot signification est tellement singulière que l'auteur lui-même, après avoir proclamé qu'un mot a toujours une seule signification, emploie à la page suivante le mot signification pas dans le sens qu'il vient de donner, mais dans son acception tout à fait usuelle (Reichling, 1961, p. 38, ligne 3). "Chassez le naturel, il revient au galop.» On ne peut s'en étonner : dans la pratique de tous les jours on parle en effet des significations du mot.

d) L'objection la plus importante que l'on peut faire contre la façon dont Reichling manie le terme signification, c'est qu'elle est difficile à manier en lexicographie synchronique et tout à fait inutilisable en lexicographie diachronique.

Le lexicographe synchronique ne peut que difficilement s'en servir, étant donné la variabilité subjective du conglomérat sémantique réuni sous ce que Reichling appelle signification. Si l'on objecte que la division du continuum sémantique en significations traditionnelles n'est pas dépourvu tout à fait de subjectivité non plus, il ne me reste qu'à m'incliner. La délimitation des significations multiples et enchevêtrées du mot est, en effet, une tâche très complexe (Ullmann, 1959, p. 97). Mais alors on peut se demander : qu'a-t-on gagné si l'on supprime pour les subdivisions sémantiques du mot le terme significations en les remplaçant par facettes ou distinctions (Reichling, 1965, p. 41) de la soidisant signification unique insaisissable? Cette «signification» unique a un niveau d'abstraction trop élevé pour qu'elle puisse servir à la discipline pratique qu'est la lexicographie.

Pour la lexicographie historique, appelée parfois «panchronique » (ReyDebove, 1971, p. 165), la terminologie reichlingienne est tout à fait impossible. En effet si c'est déjà difficile de délimiter cette signification unique d'un point de vue synchronique, il est tout à fait exclu qu'on puisse faire une délimitation pareille pour une période de temps de cinq cents, de mille ans ou plus. Si cette 
soi-disant signification unique peut de nos jours varier d'individu en individu, quel est alors le degré de variabilité inhérent à des époques d'une telle étendue ? Pour l'auteur du dictionnaire historique cette signification unique ne peut être qu'une chimère. Si le lexicographe historique se figure parfois de pouvoir constituer une chaîne de transitions sémantiques graduelles qui laisse comprendre les diverses facettes sémantiques appelées traditionnellement significations du contenu, si, en d'autres mots, il lui est possible de passer d'une signification à une autre, il ne pourra cependant décréter, pour n'importe quelle période du passé, que, par exemple, grue «oiseau échassier», grue « appareil de levage » (qui paraît calqué sur le moyen néerlandais crane) et grue «femme de mours faciles», ont formé une seule chaîne sémantique continue sur laquelle il serait possible de coller l'étiquette collectiviste de signification unique. Le lexicographe historique ne dispose point en effet d'une seconde vue.

Il y a quelques années que parut dans la revue Language un article sur la place du dictionnaire dans la description linguistique. Dans cet article, de la main de Madeleine Mathiot, on peut lire les mots suivants, - je traduis «Une définition qui veut être un instrument analytique utile, a pour condition fondamentale d'être opérationnelle. » On pourrait dire la même chose de la théorie de la signification du mot en vogue chez plusieurs linguistes hollandais : elle est non opérationnelle, donc inutilisable, en lexicographie. Ce que Weinreich disait en novembre 1960 (Weinreich, 1962, p. 28), notamment que plusieurs théories sémantiques semblent être exclues comme base de la lexicographie, s'applique aussi à la théorie de la signification du mot de Reichling.

F. DE Tollenaere

\section{BIBLIOGRAPHIE}

AlleN, W. S. (1957) : On the Linguistic Study of Languages. An Inaugural Lecture, Cambridge.

DrK, S. C., et J. G. KoorJ (1970) : Beginselen van de algemene taalwetenschap, Utrecht et Anvers.

Gleason, H. A. Jr. (1962) : "The Relation of Lexicon and Grammar ", in Fred. W. Householder et Sol Saporta, Problems in Lexicography = International Journal of American Linguistics, vol. $28, \mathrm{n}^{\circ} 2$, avril, p. 85-102.

Mathiot, M. (1967) : "The Place of the Dictionary in Linguistic Description », Language, 43 , p. $703-724$.

REICHLING, A. (1935) : Het Woord. Een studie omtrent de grondslag van taal en taalgebruik, Nijmegen.

- (1961) : Verzamelde studies over hedendaagse problemen der taalwetenschap, Zwolle.

- (1963) : «Das Problem der Bedeutung in der Sprachwissenschaft " Inssbrucker Beiträge zur Kulturwissenschaft, Sonderheft 19, Innsbruck.

- (1965) : Verzamelde studies over hedendaagse problemen der taalwetenschap. Derde herziene en uitgebreide druk, Zwolle.

- (1969) : Verzamelde studies over hedendaagse problemen der taalwetenschap, Vijfde druk, Zwolle.

REY-DEBove, J. (1971) : Etude linguistique et sémiotique des dictionnaires français contemporains, La Haye et Paris, Mouton, «Approaches to Semiotics». 
StutTerheim, C. F. P. (1941-1942) : «De polysemie der taal : een probleem der philosophische taal-critiek », Algemeen Nederlands Tijdschrift voor Wijsbegeerte en Psychologie, 35 , p. $183-199$.

Ullmann, S. (1952) : Précis de sémantique française, Berne.

- (1959) : Précis de sémantique française, Berne.

WeIENREICH, U. (1962) : "Lexicographic Definition in Descriptive Semantics», in Fred W. Householder et Sol Saporta, Problems in Lexicography = International Journal of American Linguistics, vol. $28, \mathrm{n}^{\circ} 2$, avril, p. 25-43.

Zgusta, L. (1971) : Manual of Lexicography, La Haye et Paris. 